\title{
Combining Panel Data Sets with Attrition and Refreshment Samples ${ }^{1}$
}

\author{
Keisuke Hirano - Harvard University \\ Guido W. Imbens - UCLA \\ Geert Ridder - Free University Amsterdam \\ Donald B. Rubin - Harvard University
}

February 1998

\footnotetext{
${ }^{1}$ The authors thank Joshua Angrist, Gary Chamberlain, Jerry Hausman, and participants in seminars at Harvard-MIT, NYu, UCLA and especially students at Wisconsin for comments.
} 


\begin{abstract}
With panel data important issues can be resolved that can not be addressed with cross-sectional data. A major drawback is that panel data suffer from more severe missing data problems. Adding a sample consisting of new units randomly drawn from the original population as replacements for units who have dropped out of the panel, a so-called refreshment sample, can be helpful in mitigating the effects of attrition, both by allowing for estimation of richer models and by making estimation of conventional models more precise. In this paper we develop a family of models that incorporate refreshment samples, and we demonstrate in an application to a Dutch data set on travel behaviour that such models can lead to substantially different results than models that assume that the missing data process is ignorable or conventional econometric models for panel data with attrition.
\end{abstract}

Keywords and phrases: panel data, missing data, attrition.

JEL code: C23, $C 81$. 


\section{INTRODUCTION}

In economics and other fields, researchers often wish to consider statistical models that allow for more complex relationships than can be inferred using only cross-sectional data. Panel, or longitudinal, data, in which the same units are observed repeatedly at different points in time, can often provide the richer data needed for such models (Chamberlain, 1984; Hsiao, 1986; Baltagi, 1995). Although panel data allow researchers to identify more complex models than cross-sectional data, missing data problems can be more severe in panels. In particular, even units who respond in initial waves of the panel may drop out of the sample in subsequent waves, so that the subsample with complete data for all waves of the panel can be less representative of the population than the original sample (e.g., Hausman and Wise, 1979; Ridder, 1990; Verbeek and Nijman, 1992; Abowd, Crepon, Kramarz, and Trognon, 1995).

Sometimes, in the hope of mitigating the effects of attrition without losing the fundamental advantages of panel data over cross-sections, panel data sets are augmented by replacing units who have dropped out with new units randomly sampled from the original population. Following Ridder (1992), who used these replacement units to test some models for attrition, we call such additional samples refreshment samples. In this paper we explore the benefits of refreshment samples for inference in the presence of attrition. The two themes of the paper are, first, that refreshment samples can improve inference under conventional models by providing additional sample information, and second, that refreshment samples allow for estimation of more general models of attrition without requiring auxiliary assumptions on distributions of the response variables. Thus, refreshment samples are potentially a relatively inexpensive way to improve the quality of longitudinal surveys.

In the following section we lay out the structure of the data and the inferential problem. In Section 3 we describe two conventional models for attrition in panel data. The first model, based on the missing at random assumption (MAR, Rubin, 1976; Little and Rubin, 1987), allows the probability of attrition to depend on lagged but not on contemporaneous variables. 
This case is sometimes also referred to as selection on observables (Moffitt, Fitzgerald, and Gottschalk, 1997). The second model (denoted by HW in the remainder of the paper, given its origin in a model developed by Hausman and Wise (1979)), allows the probability of attrition to depend on contemporaneous, but not lagged, variables. It is also referred to as selection on unobservables (Moffitt, Fitzgerald, and Gottschalk, 1997) because attrition partly depends on variables that are not observed if the unit drops out. In Sections 4 and 5 we present the main theoretical contributions. We develop a model for attrition that includes the MAR and HW models as special cases. This Additive Nonignorable (AN) model is identified with no testable implications given the availability of a panel data set and a refreshment sample. We first discuss in Section 4 the identification issues in a simple two-period context with a single binary variable in both periods, and generalize the model to allow for multi-valued variables as well as time-invariant covariates in Section 5.

In Section 6 we apply the ideas presented in this paper to a panel data set on travel behavior in the Netherlands, the Dutch Transportation Panel (DTP). This data set is based on a survey of Dutch households concerning their use of various modes of transportation. For a number of years households were asked to keep a detailed travel diary for an entire week each year. For every trip taken by a household member detailed information was gathered including destination, time, and mode of transportation. Attrition was severe, and because the considerable effort required to respond to the survey was directly related to the value of one of the variables (total number of trips taken by household members), it is plausible that among those who responded in the first wave the willingness to cooperate in the second wave depended on the value of these variables in either first or second period. Because MAR rules out dependence on second period variables, and the HW model rules out dependence on first period variables, this makes for a potentially interesting comparison of the performance of the MAR and HW models. In this application we implement the models by imputing the missing data according to the various missing data models, and compare the results both in terms of estimates of the relation of the change in the number of trips to the change 
in income as well as in terms of estimates of the attrition process itself. Imputation has the advantage over joint estimation of the model for attrition and the substantive model of interest that it frees the researcher from having to adopt the estimation procedures to allow for missing data. Given the complex nature of modern models for panel data (e.g., Honore, 1992; Kyriazidou, 1997), this can be a substantial benefit.

\section{The Sampling Framework}

In this section we set up the general sampling framework and discuss the identification problem. In the first period we draw a random sample of size $N_{p}$ from a fixed population. For each unit $i$, for $i=1, \ldots, N_{p}$, we observe an outcome variable $Z_{i 1}$. For a subset of this sample we observe in the second period a second variable $Z_{i 2}$; the remaining units have dropped out of the panel and all of their $Z_{i 2}$ are missing. We refer to the first subsample, of size $N_{c p}$, as the "complete panel" subsample, the second subsample, of size $N_{i p}$, as the "incomplete panel" subsample, and the two combined as the "panel", with $N_{p}=N_{c p}+N_{i p}$. In addition to the panel data set we draw in the second period a new random sample from the original population, the "refreshment" subsample, of size $N_{r}$. For these units we observe $Z_{i 2}$ but not $Z_{i 1}$. Finally, for all $N=N_{c p}+N_{i p}+N_{r}$ units we observe a vector of time-invariant covariates denoted by $X_{i}$.

We formalize the data-generating process as follows. Each unit in the population is randomly assigned a three-valued indicator $A_{i}$. If assigned $A_{i}=2$, unit $i$ is designated to be part of the panel and will be approached in both periods. If assigned $A_{i}=1$, unit $i$ is designated to be part of the refreshment sample and will be approached only in the second period. Finally, if assigned $A_{i}=0$ the unit will not be approached at all. We assume that all units respond the first time they are approached: if assigned $A_{i}=2$, unit $i$ will respond in the first period and $Z_{i 1}$ and $X_{i}$ will be recorded, and if assigned $A_{i}=1$, unit $i$ will respond in the second period and $Z_{i 2}$ and $X_{i}$ will be recorded. Not all units, however, respond the second time they are approached. In particular, not all units assigned $A_{i}=2$ will respond in 
the second period. Let $W_{i}$ be an indicator denoting the willingness to respond repeatedly; $W_{i}=1$ implies that unit $i$, if approached in the second period after already having responded in the first period, will respond, and $Z_{i 2}$ will be recorded, and $W_{i}=0$ implies that unit $i$, if approached in the second period after already having responded in the first period, will not respond, and $Z_{i 2}$ will not be recorded. The willingness to respond $W_{i}$ is observed if and only if the researcher attempts to get a second response from unit $i$, that is we observe $W_{i}$ only if $A_{i}=2$. We can use the design variable, $A_{i}$, and the willingness to respond, $W_{i}$, to define two missing data indicators $D_{i 1}$ and $D_{i 2}$. When $D_{i 1}=1$ we observe $Z_{i 1}$ and when $D_{i 1}=0$ we do not observe $Z_{i 1}$. Similarly, when $D_{i 2}=1$ we observe $Z_{i 2}$ and when $D_{i 2}=0$ we do not observe $Z_{i 2}$ :

$$
D_{i 1}=1_{A_{i}=2}, \quad \text { and } \quad D_{i 2}=1\left\{A_{i}=1\right\}+1\left\{A_{i}=2\right\} \cdot W_{i}
$$

The two missing data indicators are always observed. Table 1 illustrates the missing data pattern. Note that the missing data pattern is not monotone in the terminology of Little and Rubin (1987); for some units $Z_{i 1}$ is missing but $Z_{i 2}$ is observed whereas for others $Z_{i 2}$ is missing but $Z_{i 1}$ is observed. The missing data pattern resembles that studied in the literature on estimation of cell frequencies in contingency tables with known marginals (e.g., Little and $\mathrm{Wu}, 1991)$. The main differences are that we do now assume exact knowledge of the marginal distributions, and we allow for continuous as well as multinomimal variables.

Table 1: Missing Data Pattern: Observed $(\times)$ or Missing $(-)$, or Actual Values $(0,1$, OR 2$)$

\begin{tabular}{cc|c|cccc} 
Missing Data Indicators & Design Variable & \multicolumn{5}{|c}{ Individual Characteristics } \\
$D_{i 1}$ & $D_{i 2}$ & $A_{i}$ & $W_{i}$ & $Z_{i 1}$ & $Z_{i 2}$ & $X_{i}$ \\
\hline 0 & 0 & 0 & - & - & - & - \\
0 & 1 & 1 & - & - & $\times$ & $\times$ \\
1 & 1 & 2 & 1 & $\times$ & $\times$ & $\times$ \\
1 & 0 & 2 & 0 & $\times$ & - & $\times$
\end{tabular}


The design variable $A_{i}$ is under control of the surveyor, and by assumption it satisfies the following independence condition: ${ }^{1}$

$$
A_{i} \perp W_{i}, Z_{i 1}, Z_{i 2}, X_{i}
$$

In the remainder of this section we make some comments on the sampling framework and provide some motivation for the focus of the paper.

First consider the willingness to respond repeatedly $W_{i}$, or its complement, the attrition indicator $1-W_{i}$. It is interpreted as an individual characteristic. It has the unusual feature that it cannot be directly measured. It can only be revealed by actions of the surveyor: by approaching a person in the first period, that is by assigning unit $i$ the value $A_{i}=2$, this willingness to respond gets revealed in the second period. It is also important to stress again that there is no intrinsic interest in its distribution. The substantive interest is in the joint distribution of $\left(Z_{i 1}, Z_{i 2}, X_{i}\right)$, or possibly the conditional distribution of $\left(Z_{i 1}, Z_{i 2}\right)$ given $X_{i}$. The distribution of the willingness to respond is of concern solely because its properties might affect our ability to recover the distributions of interest.

A second issue is that of conventional non-response problems. We assume throughout the analysis that we always oberve $Z_{i 1}$ if we assign $A_{i}=2$ to unit $i$, and similarly we always observe $Z_{i 2}$ if we assign $A_{i}=1$ to unit $i$. There might, however, be non-response of the standard cross-section type present, where we know nothing about units other than that they did not respond, or where we know some variables but not others for some units. Ridder (1992) discusses these issues for the particular data set we use in this paper. We ignore such issues here to focus on the specific panel-data problem of attrition of units who are initially prepared to respond but choose not to do so in subsequent waves of the panel.

\footnotetext{
${ }^{1}$ In fact this independence condition is stronger than necessary. In practice we are primarily interested in the conditional distribution of $\left(Z_{i 1}, Z_{i 2}\right)$ given $X_{i}$ rather than the joint distribution of $\left(Z_{i 1}, Z_{i 2}, X_{i}\right)$, and for features of this distribution it suffices that$$
A_{i} \perp W_{i}, Z_{i 1}, Z_{i 2} \mid X_{i},
$$
combined with positive probabilities for all values of $A_{i}$ conditional on $X_{i}$.
} 
A third issue is the focus on the specification of the attrition probability

$$
\operatorname{Pr}\left(W_{i}=1 \mid Z_{i 1}, Z_{i 2}, X_{i}\right)
$$

To justify this focus, consider the identification of the joint distribution of $\left(Z_{i 1}, Z_{i 2}, X_{i}\right)$. By definition,

$$
f\left(Z_{i 1}, Z_{i 2}, X_{i}\right)=\frac{f\left(Z_{i 1}, Z_{i 2}, X_{i} \mid W_{i}=1\right) \cdot \operatorname{Pr}\left(W_{i}=1\right)}{\operatorname{Pr}\left(W_{i}=1 \mid Z_{i 1}, Z_{i 2}, X_{i}\right)} .
$$

Because $A_{i} \perp W_{i}, Z_{i 1}, Z_{i 2}, X_{i}$, the two factors in the numerator of the righthand side can be rewritten as

$$
f\left(Z_{i 1}, Z_{i 2}, X_{i} \mid W_{i}=1\right)=f\left(Z_{i 1}, Z_{i 2}, X_{i} \mid W_{i}=1, A_{i}=2\right)=f\left(Z_{i 1}, Z_{i 2}, X_{i} \mid D_{i 1}=1, D_{i 2}=1\right),
$$

and

$$
\operatorname{Pr}\left(W_{i}=1\right)=\operatorname{Pr}\left(W_{i}=1 \mid A_{i}=1\right)=\operatorname{Pr}\left(D_{i 2}=1 \mid D_{i 1}=1\right),
$$

both of which can be estimated directly from the panel data set. Identification of the attrition probability $\operatorname{Pr}\left(W_{i}=1 \mid Z_{i 1}, Z_{i 2}, X_{i}\right)$ therefore ensures identification of the joint distribution of $\left(Z_{i 1}, Z_{i 2}, X_{i}\right)$.

A fourth comment concerns inference given a model for attrition. Given knowledge of the conditional probability $\operatorname{Pr}\left(W_{i}=1 \mid Z_{i 1}, Z_{i 2}, X_{i}\right)$ inference can proceed in different ways. One can use the inverse of this conditional probability to weight the complete panel, that is, the observations with $A_{i}=2$ and $W_{i}=1$, and use weighted versions of the complete data estimation techniques (e.g., Hansen, Hurwitz and Madow, 1966; Imbens and Hellerstein, 1994; Nevo, 1995). Alternatively one can use this conditional probability to impute the missing values and use complete data estimation techniques on the imputed data sets (Rubin, 1987, 1996). See Brownstone and Valetta (1995) for a recent economic application. In the application in Section 6 we use the second of these approaches. A major reason is that the theoretical models we develop in Sections 4 and 5 are conveniently implemented using 
the Data Augmentation (DA) algorithm proposed by Tanner and Wong (1987), which as a by-product generates imputed data sets that allow for standard complete data estimation. ${ }^{2}$

\section{Models for Panel Data with Attrition}

In this section we review two models that have been proposed in the literature to address the problem of attrition in panel data. In terms of the notation defined in the previous section, we only have the subsample with $A_{i}=2$. In Section 4.1 we shall see that these models employ assumptions that, although to some extent unavoidable in the context for which they were designed, can substantially be relaxed in the presence of refreshment samples. In the application in Section 6 we shall evaluate the appropriateness of these assumptions for the particular data set analyzed.

\subsection{Missing at Random (MAR)}

The first model makes the assumption that $Z_{i 2}$ is missing at random (MAR) in the panel,

$$
W_{i} \perp Z_{i 2} \mid Z_{i 1}, X_{i} \quad(\mathrm{MAR}),
$$

implying that if all the parameters of the missing data process are distinct from those of the data distribution, and the probability of $W_{i}=1$ is greater than zero, then the missing data process is ignorable (Rubin, 1976; Little and Rubin, 1987).

The special case arising when

$$
W_{i} \perp Z_{i 1}, Z_{i 2}, X_{i} \quad(\mathrm{MCAR})
$$

is referred to as missing completely at random (MCAR). In that case no bias results from limiting the analysis to the complete panel with $D_{i 1}=D_{i 2}=1$.

\footnotetext{
${ }^{2}$ The imputation approach is particularly convenient for our application because it simultaneously allows us to deal with the fact that one of the variables, income, is only observed to lie in one of four intervals. This creates complications even in the absence of attrition if we wish to regress the level of income on another variable. Here we impute the level of income as part of the general imputation procedure.
} 


\subsection{The Hausman-Wise (HW) model for Attrition}

The second model for panel data with attrition we consider is a generalization of a model developed by Hausman and Wise (1979), and is related to models developed for crosssectional surveys by Heckman (1979). Hausman and Wise allow the probability of attrition in the second period to depend on the contemporaneous, that is second period, variables $Z_{i 2}$ but assume that the first period variables do not affect this probability:

$$
W_{i} \perp Z_{i 1} \mid Z_{i 2}, X_{i} \quad(\mathrm{HW}) .
$$

The original formulation of the Hausman-Wise model (Hausman and Wise, 1991) also restricts the joint distribution of $Z_{i 1}$ and $Z_{i 2}$ and assumes normality of some of the variables, but these restrictions can partly be relaxed and do not concern us here. The appeal of these models is that they can reflect optimal behavior of the respondent whose effort in responding is related to the anticipated value of $Z_{i 2}$. An implication is that the distribution of $Z_{i 2}$ given $\left(Z_{i 1}, X_{i}\right)$ for those with $W_{i}=0$ differs in a systematic way from the distribution for those with $W_{i}=1$. Again the MCAR case is a special case of this attrition model. In a conventional panel survey with no refreshment samples, neither MAR or HW are testable without auxiliary assumptions.

\section{A Simple Example with Binary Variables}

In this section we assume that $Z_{i 1}$ and $Z_{i 2}$ are binary variables and suppress the conditioning on time-invariant covariates $X_{i}$. Denote the conditional probability $\operatorname{Pr}\left(Z_{i 2}=1 \mid Z_{i 1}=\right.$ $\left.z, W_{i}=w\right)$ by $q_{z w}$, and the probability $\operatorname{Pr}\left(Z_{i 1}=z, W_{i}=w\right)$ by $r_{z w}$. In large samples we can learn the value of $r_{z w}$ for $z, w \in\{0,1\}$ because the subsample with $A_{i}=2$ is a random sample from the population, and for this subsample we always observe $Z_{i 1}$ and $W_{i}$. Similarly we can learn in large samples the values of $q_{z 1}$ for $z=0,1$, because $A_{i} \perp\left(W_{i}, Z_{i 1}, Z_{i 2}\right)$ implies that the subsample with $A_{i}=2, W_{i}=1$ and $Z_{i 1}=z$ is a random sample from the subpopulation with $W_{i}=1$ and $Z_{i 1}=z$, and for this subsample we always observe $Z_{i 2}$. The 
data with $A_{i}=2$, however, contain no information on $q_{z 0}$ because we never observe $Z_{i 2}$ if $W_{i}=0$ and $A_{i}=2$.

The subsample with $A_{i}=1$ allows us to deduce in large samples the marginal distribution of $Z_{i 2}$. Since

$$
\operatorname{Pr}\left(Z_{i 2}=1\right)=\sum_{z, w} q_{z w} \cdot r_{z w}
$$

knowledge of marginal distribution of $Z_{i 2}$ implies a single linear restriction on the two remaining parameters $q_{10}$ and $q_{00}$ in terms of the directly estimable parameters $q_{01}, q_{11}$ and $r_{00}$, $r_{01}, r_{10}$, and $r_{11}$. The panel and refreshment sample combined do therefore not enable us to estimate the values of $q_{00}$ and $q_{10}$ uniquely from the population distribution of the observed data, although they do imply a linear restriction on these two parameters.

\subsection{Testable Implications of the MaR and HW Models in the Presence of REFRESHMENT SAMPLES}

The MAR and HW models do not require the refreshment sample for estimation of $q_{00}$ and $q_{10}$. The independence assumptions (3) and (4) each imply two restrictions on the eight parameters $r_{z w}$ and $q_{z w}$ that are sufficient for identication of $q_{00}$ and $q_{10}$. Specifically, MAR implies

$$
q_{00}=q_{01}
$$

and

$$
q_{10}=q_{11}
$$

Under the HW assumption the relation is more complex, but it can be shown to imply

$$
q_{00}=\frac{r_{10} \cdot r_{01} \cdot\left(1-q_{01}\right)-r_{11} \cdot r_{00} \cdot\left(1-q_{11}\right)}{r_{00} \cdot r_{11} \cdot q_{11} \cdot\left(1-q_{01}\right) / q_{01}-r_{11} \cdot r_{00} \cdot\left(1-q_{11}\right)},
$$

and

$$
q_{10}=\frac{q_{00} \cdot r_{00} \cdot q_{11} \cdot r_{11}}{q_{01} \cdot r_{01} \cdot r_{10}}
$$


Under either of these two models, therefore, we can estimate all eight parameters from the panel alone. In each case these estimates imply a marginal distribution for $Z_{i 2}$. This distribution can be compared to the distribution of $Z_{i 2}$ in the refreshment sample to test the attrition model that generated it.

To illustrate these issues we use in this section a subset of the data set that will be analysed in more detail in Section 5. We define a binary variable indicating whether the total number of trips for a household during the survey week was less than or equal to twenty-five.

Tables 2 summarizes the sample information for this variable and Table 3 presents estimates of the six parameters that are directly estimable from the panel data alone as well as estimates for $q_{00}$ and $q_{10}$ under the MAR and HW assumptions.

Table 2: Summary Statistics Dutch Transportation Panel: $Z_{i t}$ Is indicator for NUMBER OF TRIPS IN THE PERIOD $t$ LESS THAN OR EQUAL TO 25, AND $W_{i}$ IS INDICATOR FOR WILLINGNESS TO RESPOND IN THE SECOND PERIOD.

$$
\text { Subsample } \quad Z_{i 1} \quad Z_{i 2} \quad W_{i} \quad \text { No of obs }
$$

\begin{tabular}{ccccc}
\hline Complete Panel & 0 & 0 & 1 & 832 \\
& 0 & 1 & 1 & 66 \\
& 1 & 0 & 1 & 53 \\
& 1 & 1 & 1 & 80 \\
Incomplete Panel & 0 & - & 0 & 518 \\
& 1 & - & 0 & 215 \\
Refreshment Sample & - & 0 & - & 520 \\
& - & 1 & - & 136
\end{tabular}

Assuming MAR, the panel subsample with $A_{i}=2$ leads to the estimates $\hat{q}_{00}=\hat{q}_{01}=$ 0.074 , and $\hat{q}_{10}=\hat{q}_{11}=0.602$, which in turn implies that the marginal probability of the number of trips in the second period exceeding twenty-five is $\hat{r}_{00} \cdot \hat{q}_{00}+\hat{r}_{01} \cdot \hat{q}_{01}+\hat{r}_{10}$. 
Table 3: Estimates Based on the COMPlete PAnel subsample

\begin{tabular}{c|ccccc} 
& \multicolumn{2}{|c}{ Directly Estimable Parameters } & \multicolumn{2}{c}{ Model-based Estimates } \\
& $W_{i}=0$ & $W_{i}=1$ & $q_{z 1}$ & MAR & HW \\
$Z_{i 1}=0$ & $\hat{r}_{00}=0.294$ & $\hat{r}_{01}=0.509$ & $\hat{q}_{01}=0.074$ & $\hat{q}_{00}=0.074$ & $\hat{q}_{00}=0.306$ \\
$Z_{i 1}=1$ & $\hat{r}_{10}=0.122$ & $\hat{r}_{11}=0.075$ & $\hat{q}_{11}=0.602$ & $\hat{q}_{10}=0.602$ & $\hat{q}_{10}=0.894$
\end{tabular}

$\hat{q}_{10}+\hat{r}_{11} \cdot \hat{q}_{11}=0.178$. This differs from the marginal probability of the number of trips in the second period exceeding twenty-five implied by the refreshment sample, which is $136 /(136+520)=0.207$. A likelihood ratio test, however, with a nominal $\mathcal{X}^{2}(1)$ distribution, gives a test-statistic of 2.2, shows that this is not statistically significant at conventional levels.

Assuming HW and again ignoring sampling error, the two proportions that cannot directly be estimated from the data are

$$
\begin{aligned}
& \hat{q}_{00}=\frac{\hat{r}_{10} \cdot \hat{r}_{01} \cdot\left(1-\hat{q}_{01}\right)-\hat{r}_{11} \cdot \hat{r}_{00} \cdot\left(1-\hat{q}_{11}\right)}{\hat{r}_{00} \cdot \hat{r}_{11} \cdot \hat{q}_{11} \cdot\left(1-\hat{q}_{01}\right) / \hat{q}_{01}-\hat{r}_{11} \cdot \hat{r}_{00} \cdot\left(1-\hat{q}_{11}\right)}=0.306, \\
& \hat{q}_{10}=\frac{\hat{q}_{00} \cdot \hat{r}_{00} \cdot \hat{q}_{11} \cdot \hat{r}_{11}}{\hat{q}_{01} \cdot \hat{r}_{01} \cdot \hat{r}_{10}}=0.894,
\end{aligned}
$$

leading to an estimate for the marginal probability of the number of trips in the second period being less than or equal to twenty-five of 0.282 , substantially different from the refreshment sample estimate of 0.207 . A likelihood ratio test gives 7.8 , with a nominal $\mathcal{X}^{2}(1)$, statistically significant at at the 0.05 level.

\subsection{The Additive Nonignorable (AN) Model}

The above discussion demonstrates that MAR and HW models have testable implications if refreshment samples are available, suggesting that more general models may be identified. We therefore proceed to develop a model that generalizes MAR and HW in a way that has 
no testable implications. Suppose we model, with no essential loss of generality ${ }^{3}$ given the binary nature of $Z_{i 1}$ and $Z_{i 2}$, the probability of response as

$$
\operatorname{Pr}\left(W_{i}=1 \mid Z_{i 1}=z_{1}, Z_{i 2}=z_{2}\right)=g\left(\alpha_{0}+\alpha_{1} \cdot z_{1}+\alpha_{2} \cdot z_{2}+\alpha_{3} \cdot z_{1} \cdot z_{2}\right),
$$

for some known, increasing $g(\cdot)$ satisfying $\lim _{a \rightarrow-\infty} g(a)=0, \lim _{a \rightarrow \infty} g(a)=1$. With $Z_{i 1}$ and $Z_{i 2}$ binary this saturates the model, implying that the choice of $g(\cdot)$ is irrelevant, and the model places essentially no restrictions on the data-generating process. Assuming MAR (HW) in this context amounts to assuming $\alpha_{2}=\alpha_{3}=0\left(\alpha_{1}=\alpha_{3}=0\right)$, and in each case the choice of $g(\cdot)$ is irrelevant.

Without restrictions on $\alpha$ the model in (5) is saturated. The discussion in the introduction to Section 4 therefore implies that this model is not identified, and we cannot estimate all four parameters $\alpha_{1}, \alpha_{2}, \alpha_{3}$, and $\alpha_{4}$ consistently from a random sample of $\left(D_{i 1} \cdot Z_{i 1}, D_{i 2}\right.$. $\left.Z_{i 2}, D_{i 1}, D_{i 2}\right)$ from the population. Figure 1 illustrates this issue in $\left(q_{00}, q_{10}\right)$ space for the data from Table 2. All values of $\left(q_{00}, q_{10}\right)$ between zero and one are consistent with the panel data. For a given $g(\cdot)$, every point $\left(q_{00}, q_{10}\right)$, combined with the data in Table 2 corresponds to a unique set of values for $\left(\alpha_{0}, \alpha_{1}, \alpha_{2}, \alpha_{3}\right)$ in (5). The " $\times$ " indicates the MAR point $\left(q_{00}, q_{10}\right)=(0.074,0.602)$, where $\alpha_{2}=\alpha_{3}=0$. The "+" indicates the HW point $\left(q_{00}, q_{10}\right)=(0.306,0.894)$ where $\alpha_{1}=\alpha_{3}=0$. Finally, the solid line indicates the set of $\left(q_{00}, q_{10}\right)$ that are consistent with $\operatorname{Pr}\left(Z_{i 2}=1\right)=0.207$ given the values of the directly estimable parameters.

Figure 1 illustrates the main issues of the paper. The most general model, not restricting the $\alpha$ 's, corresponds to the entire $\left(q_{00}, q_{10}\right)$ space. Given point estimates of the directly estimable parameters, a model, specified in terms of restrictions on the conditional probability $\operatorname{Pr}\left(W_{i}=1 \mid Z_{i 1}=z_{1}, Z_{i 2}=z_{2}\right)$, corresponds to a point or set of points in $\left(q_{00}, q_{10}\right)$ space. For example the MAR model that specifies $\left(\operatorname{Pr}\left(W_{i}=1 \mid, Z_{i 1}=z, Z_{i 2}=1\right)=\operatorname{Pr}\left(W_{i}=\right.\right.$ $1 \mid Z_{i 1}=z, Z_{i 2}=0$ ), or, equivalently, $\alpha_{2}=\alpha_{3}=0$ in (5), corresponds to the point marked

\footnotetext{
${ }^{3}$ Other than that we require the attrition probability to be strictly between zero and one.
} 
by " $\times$ ". Our approach can now be described as follows. We wish to develop models that satisfy two conditions. First, the model should always be consistent with the data, which in terms of this figure means that the intersection of the set of $\left(q_{00}, q_{10}\right)$ consistent with the model and the set of points on the solid line (i.e., the set of points that corresponds to the marginal distribution for $Z_{i 2}$ estimated from the refreshment sample) is nonempty for any set of observed values of $\hat{q}_{z w}$ and $\hat{r}_{z w}$. Second, the model should be identified. That is, this intersection should contain only a single point. The models imposing MAR or HW a priori fail the first condition because they are not always consistent with the data, and the general unrestricted model fails the second because it is not identified.

The alternative family of models we suggest has the form

$$
\operatorname{Pr}\left(W_{i}=1 \mid Z_{i 1}=z_{1}, Z_{i 2}=z_{2}\right)=g\left(\alpha_{0}+\alpha_{1} \cdot z_{1}+\alpha_{2} \cdot z_{2}\right),
$$

for unrestricted values of the unknown parameters $\alpha_{0}, \alpha_{1}$, and $\alpha_{2}$. This model rules out an interaction term between $Z_{i 1}$ and $Z_{i 2}$, but allows for non-ignorable models by allowing $\alpha_{2}$ to differ from zero. To reflect the additivity of the index in the $g(\cdot)$ function in first and second period variables we refer to this as the additive non-ignorable (AN) model. Note that both the MAR and HW models are special cases of this model.

In Figure 2 we illustrate, for the case of a logistic $g(a)=\exp (a) /(1+\exp (a))$, the set of points consistent with both the model and the directly estimable $q_{z w}$ and $r_{z 1}$ (the solid curve). Note that there is a single point of intersection between this set and the set of points consistent with the marginal distribution of $Z_{i 2}$ (solid line). Also note that the set of points consistent with the model includes both the MAR and HW points. The latter is trivial because the MAR (HW) point corresponds to $\alpha_{2}=0\left(\alpha_{1}=0\right)$ in the model. Both points are true in general, irrespective of the choice of $g(\cdot)$ and the population distributions, as will be shown in the following theorem.

Theorem 1 For any quadruple $q_{01}, q_{11} \in(0,1)$, any quadruple $r_{z w} \in(0,1)$ with $\sum_{z w} r_{z w}=1$, and any continuous and increasing $g(\cdot)$ with $\lim _{a \rightarrow-\infty} g(a)=0$ and $\lim _{a \rightarrow \infty} g(a)=1$, there 
is a unique quintuple $\left(\alpha_{0}, \alpha_{1}, \alpha_{2}, \hat{q}_{00}, \hat{q}_{10}\right)$ with $\hat{q}_{00}, \hat{q}_{10} \in(0,1)$ such that the following five conditions are satisfied:

$$
\begin{aligned}
& g\left(\alpha_{0}\right)=\left(1-q_{01}\right) r_{01} /\left(\left(1-q_{01}\right) r_{01}+\left(1-\hat{q}_{00}\right) r_{00}\right), \\
& g\left(\alpha_{0}+\alpha_{1}\right)=\left(1-q_{11}\right) r_{11} /\left(\left(1-q_{11}\right) r_{11}+\left(1-\hat{q}_{10}\right) r_{10}\right), \\
& g\left(\alpha_{0}+\alpha_{2}\right)=q_{01} r_{01} /\left(q_{01} r_{01}+\hat{q}_{00} r_{00}\right), \\
& g\left(\alpha_{0}+\alpha_{1}+\alpha_{2}\right)=q_{11} r_{11} /\left(q_{11} r_{11}+\hat{q}_{10} r_{10}\right),
\end{aligned}
$$

and

$$
\hat{q}_{00} r_{00}+\hat{q}_{10} r_{10}+q_{01} r_{01}+q_{11} r_{11}=q_{00} r_{00}+q_{10} r_{10}+q_{01} r_{01}+q_{11} r_{11}
$$

Proof: See Appendix A.

An important implication of our approach is that the solutions $\hat{q}_{10}$ and $\hat{q}_{00}$ depend on the choice of $g(\cdot)$ function. Every $g(\cdot)$ function corresponds in Figure 2 to a curve approaching $(0,0)$, going through both the MAR and HW points, and approaching $(1,1)$. Nevertheless the exact point of intersection with the set of points corresponding to the restriction from the marginal distribution of $Z_{i 2}$ will in general depend on the choice of $g(\cdot)$. This differs qualitatively from both the MAR and HW models where the functional form of the selection probability is immaterial. For example, if the probability of attrition does not depend on $Z_{i 2}, g(\cdot)$ cancels from the restrictions in equations (7)-(10). The question arises how sensitive the results are to alternative choices of $g(\cdot)$. We therefore estimate the same model using a normal distribution function, or $\left.g(a)=\Phi(a)=\int_{-\infty}^{a}(1 / \sqrt{(} 2 \pi)\right) \exp \left(-z^{2} / 2\right) d z$. The dashed curve in Figure 2 illustrates the resulting set of points consistent with the panel data and the probit version of the AN model. It is clear that there is very little difference between the logit and the probit model. This is not surprising given that both approach the points $(0,0)$ and $(1,1)$, as well as go through the MAR and HW points. The difference between the two 
models around the intersection with the set of points consistent with the refreshment sample is so small that in Figure 3 we enlarge the area in the rectangle around this intersection in Figure 2.

\subsection{Estimates in the Binary Case}

Now let us return to the binary data example and consider estimation of the joint distribution of $\left(Z_{i 1}, Z_{i 2}\right)$ using the four different models and different combinations of data. For ease of exposition we focus on a single feature of this distribution

$$
\operatorname{Pr}\left(Z_{i 1}=0, Z_{i 2}=1\right)=r_{00} q_{00}+r_{01} q_{01}
$$

The rows in Table 4 correspond to the different models for attrition. The first row is based on the MCAR assumption. The second row is based on the MAR assumption. The third row presents estimates based on the HW model, and the next two rows are based on the AN model, using the logistic and normal distribution function for $g(\cdot)$. The different columns correspond to different data sets. The first column presents estimates based on the complete panel data set alone (observations with $A_{i}=2$ and $W_{i}=1$ ). Only the MCAR model is identified in that case, so only estimates for this model are reported. The next column presents estimates based on the panel data alone (all observations with $A_{i}=2$ ). Now the HW and MAR models are identified as well, so estimates are reported for the first three models. Finally, the last column reports estimates based on all observations. In this case all models are identified (MCAR, MAR, and HW are in fact overidentified), and estimates are reported for all five models, MCAR, MAR, HW, and the logit and probit versions of AN.

In the last row we also report nonparametric bounds on the probability of $\operatorname{Pr}\left(Z_{i 1}=\right.$ $0, Z_{i 2}=1$ ), in the spirit of work by Manski (1995) and Horowitz and Manski (1997). These bounds demonstrate the identifying power of the various parts of the data set. In the first set of bounds on $\operatorname{pr}\left(Z_{i 1}=0, Z_{i 2}=1\right)=r_{00} q_{00}+r_{01} q_{01}$ we assume that we have knowledge of the complete panel subsample and the frequency of attrition. This allows us to estimate $r_{01}$ and $q_{01}$. Nothing is known about $q_{00}$ (other than that it is between zero and one) and $r_{00}$ is 
only known to lie in the interval $\left(0, \hat{r}_{00}+\hat{r}_{10}\right)=(0,0.416)$. In the second set of bounds we add the incomplete panel subsample. This allows us to estimate $r_{00}$ as 0.294 but still nothing is known about $q_{00}$. In the last set of bounds we add the refreshment sample. This narrows down the interval for $q_{00}$ from $(0,1)$ to $(0.007,0.422)$. In each case the bounds narrow with the additional information.

Table 4: Maximum Likelihood Estimates of $\operatorname{Pr}\left(Z_{i 1}=0, Z_{i 2}=1\right)$

\begin{tabular}{lc|ccc} 
Model & $g(\cdot)$ & Complete Panel & Panel & Panel and Refreshment Sample \\
& & $\left(A_{i}=2, W_{i}=1\right)$ & $\left(A_{i}=2\right)$ & $\left(A_{i}=1,2\right)$ \\
\hline MCAR & & 0.051 & 0.079 & 0.070 \\
MAR & & 0.079 & 0.075 \\
HW & & 0.043 & 0.048 \\
AN & logit & & & 0.073 \\
AN & probit & & & 0.073 \\
Bounds & & $(0.038,0.454)$ & $(0.038,0.332)$ & $(0.040,0.162)$
\end{tabular}

An important feature of Table 4 is that whatever the model, MCAR, MAR, HW or AN, the differences generally decrease in magnitude the more data are used. Consider for example the differences between the HW and MAR estimates of $\operatorname{Pr}\left(Z_{i 1}=0, Z_{i 2}=1\right)$ based on panel data alone: 0.043 and 0.079 respectively. The estimates are considerably closer when we also use the refreshment sample: 0.048 and 0.075, and both are closer to the AN estimates of 0.073. The exact difference between the probit and logit version of the AN model are extremely small: 0.07341 for the logit version, and 0.07339 for the probit version. In the binary case the estimates based on the MAR and HW models do not depend on the choice of $g(\cdot)$. 


\section{Identification With Multi-VAlued and Time-Invariant Variables}

In this section we generalize the identification result in Section 4.3 to allow for multivalued and time-invariant variables.

Theorem 2 Let $f\left(z_{1}, z_{2}, x\right)$ be the joint probability function of $\left(Z_{i 1}, Z_{i 2}, X_{i}\right)$, and let $p\left(z_{1}, z_{2}, x\right)$ be the conditional probability that $W_{i}=1$ given $\left(Z_{i 1}, Z_{i 2}, X_{i}\right)$, and let $0<p\left(z_{1}, z_{2}, x\right)<$ 1 for all $\left(z_{1}, z_{2}, x\right)$ in the support of $\left(Z_{i 1}, Z_{i 2}, X_{i}\right)$. Let $\left\{\left(z_{1 k}, z_{2 k}\right)\right\}_{k=1}^{K}$ be the support of $\left(Z_{i 1}, Z_{i 2}\right)$. Finally, let $g(\cdot)$ be a continuous, increasing function with $\lim _{a \rightarrow-\infty} g(a)=0$, and $\lim _{a \rightarrow \infty} g(a)=1$.

Then there is a unique set of functions $\hat{f}\left(z_{1}, z_{2}, x\right), k_{0}(x), k_{1}(z, x)$ and $k_{2}(z, x)$ such that for some $\left(\bar{z}_{1}, \bar{z}_{2}\right)$ in the support of $\left(Z_{i 1}, Z_{i 2}\right)$ :

(i), $k_{1}\left(\bar{z}_{1}, x\right)=0, k_{2}\left(\bar{z}_{2}, x\right)=0$,

(ii), $\sum_{z_{2}} \hat{f}\left(z_{1}, z_{2}, x\right)=\sum_{z_{2}} f\left(z_{1}, z_{2}, x\right)$,

(iii), $\sum_{z_{1}} \hat{f}\left(z_{1}, z_{2}, x\right)=\sum_{z_{1}} f\left(z_{1}, z_{2}, x\right)$, (iv),

$$
\hat{f}\left(z_{1}, z_{2}, x\right)=f\left(z_{1}, z_{2}, x\right) \cdot \frac{g\left(k_{0}(x)+k_{1}\left(z_{1}, x\right)+k_{2}\left(z_{2}, x\right)\right)}{p\left(z_{1}, z_{2}, x\right)} .
$$

Proof: see Appendix A

The theorem implies that given any joint distribution of $\left(Z_{i 1}, Z_{i 2}, X_{i}\right)$, and given any attrition probability $p\left(z_{1}, z_{2}, x\right)$, we can find a joint distribution of $\left(Z_{i 1}, Z_{i 2}, X_{i}\right)$ with the additive nonignorable attrition model that is "observationally equivalent". That is, take a distribution $f\left(z_{1}, z_{2}, x\right)$, and an attrition probability $p\left(z_{1}, z_{2}, x\right)$. Then we can find another distribution $\hat{f}\left(z_{1}, z_{2}, x\right)$ and another attrition probability function $\hat{p}\left(z_{1}, z_{2}, x\right)$ that leads to the same directly estimable distributions. This means the implied joint distribution of $\left(Z_{i 1}, X_{i}\right)$ is the same:

$$
\sum_{z_{2}} \hat{f}\left(z_{1}, z_{2}, x\right)=\sum_{z_{2}} f\left(z_{1}, z_{2}, x\right)
$$


In addition the joint distribution of $\left(Z_{i 2}, X_{i}\right)$ is the same:

$$
\sum_{z_{1}} \hat{f}\left(z_{1}, z_{2}, x\right)=\sum_{z_{1}} f\left(z_{1}, z_{2}, x\right)
$$

Finally, the conditional distribution given $W_{i}=1$ is the same:

$$
\frac{\hat{f}\left(z_{1}, z_{2}, x\right) \cdot \hat{p}\left(z_{1}, z_{2}, x\right)}{\sum_{z_{1}, z_{2}, x} \hat{f}\left(z_{1}, z_{2}, x\right) \cdot \hat{p}\left(z_{1}, z_{2}, x\right)}=\frac{f\left(z_{1}, z_{2}, x\right) \cdot p\left(z_{1}, z_{2}, x\right)}{\sum_{z_{1}, z_{2}, x} f\left(z_{1}, z_{2}, x\right) \cdot p\left(z_{1}, z_{2}, x\right)} .
$$

At this point there are clearly many such distributions $\hat{f}(\cdot)$ and attrition probabilities $\hat{p}(\cdot)$, including the distribution that generated the data. The theorem implies, however, that we can find a solution that imposes a particular structure on $\hat{p}(\cdot)$, namely that it can be written as additive in $z_{1}$ and $z_{2}: \hat{p}\left(z_{1}, z_{2}, x\right)=g\left(k_{0}(x)+k_{1}\left(z_{1}, x\right)+k_{2}\left(z_{2}, x\right)\right)$ for the given choice of $g(\cdot)$. Because we can do this irrespective of the original distribution $f\left(z_{1}, z_{2}, x\right)$ and the attrition probability $p\left(z_{1}, z_{2}, x\right)$, the theorem implies that the model has no testable implications, unlike the MAR and HW models.

In addition the theorem implies that this solution is unique, or that the model is identified.

Note that condition $(i)$ is a normalization that is required because of the inclusion of a constant in the additive nonignorable model. The fact that the functions $k_{1}$ and $k_{2}$ are unrestricted, makes it impossible to identify the distribution function $g(\cdot)$. It is well-known that any probability model for a binary dependent variable can be expressed as a logit by choosing an appropriate functional form for the dependence on the explanatory variables. The same is true in the AN model. Restricting these functions imposes restrictions on $g(\cdot)$.

\section{Travel Behaviour in The Netherlands}

We apply the models discussed in Sections 3 through 5 to a data set on travel behavior in the Netherlands. First we describe the data. Then we specify a fully parametric model. Given the parametric model we impute the missing data. Finally we repeatedly estimate the substantive model on the complete data sets and average the estimates to get the reported estimates. 
Although the identification results in the previous section are nonparametric in the sense that no distributional assumptions are made concerning the conditional distribution of $\left(Z_{i 1}, Z_{i 2}\right)$ given $X_{i}$, and no assumptions are made regarding the functional form of $k_{0}(x)$, $k_{1}(x, z)$ and $k_{2}(x, z)$, we shall make make such assumptions here. The reason is that with a finite sample, estimates without such smoothing assumptions would be likely to have poor sampling properties. Since we are only using the parametric model to impute missing values, and since the nonparametric identification result of the previous section ensures that the imputation is not being solely driven by the parametric form, we do not believe that much is lost in practice. In a large enough sample the parametric restrictions could be tested by classical methods, or nonparametric imputation could be carried out.

\subsection{The DATA}

The DTP collected information regarding travel behavior for six years. The households in the first wave were interviewed in March 1984, just before an increase in the price of public transportation, and the last wave was interviewed in March 1989. Households were approached twice a year, in the spring and in the fall. The spring waves involved face-to-face interviews, and the fall waves were postal surveys. We use data from the spring waves of 84 and 85, and, except for this discussion of the data, we refer to these waves as the first and second wave. ${ }^{4}$ Of the sample of 6128 households that were selected for the first wave, 2886 (47\%) agreed to participate in the panel. In the sequel we ignore potential biases induced by initial nonresponse. Of these households, 2185 were approached for an interview, and 1764 $(81 \%)$ of these provided the required information. The main purpose of the survey was to collect detailed information on household travel demand. Every household member over 11 years of age was asked to keep a travel diary, in which he/she reported all trips during a particular week. A trip starts when a household member leaves the home, and it ends upon

\footnotetext{
${ }^{4}$ The households in the first wave were obtained by a stratified sample in 20 municipalities of varying sizes. The municipalities were selected on the basis of the number of inhabitants and the availability of different types of public transportation. The sample was stratified by household type (combination of age of head and household composition), and net household income.
} 
return. In the sequel we concentrate on the total number of trips made by all household members in the survey week.

The DTP has been plagued by heavy attrition. Only $38 \%$ of the original sample participated in all seven waves of the panel. The attrition after the first wave was $41 \%$. It is not unusual that the attrition rate is the highest in the second wave of a panel study. However, in the DTP the design of the second (fall) wave increased the attrition after the first wave. In the fall wave of 1984, which was a postal survey, household members were asked to keep a travel diary. A substantial fraction $(21 \%)$ of the households did respond to this request and were dropped from the panel. In later fall waves a travel diary was not asked for. If we correct for this additional attrition, the attrition rate in wave 3 is about $20 \%$, which is about the same as the attrition observed after the first wave in the refreshment samples. In the third (spring 85) wave a refreshment sample of 656 households was added to the panel survey $^{5}$

In this paper we only use the first two spring waves. Table 5 gives the some summary statistics for the subsamples defined by the value of the design variable $A_{i}$, the willingness to participate in the second period, $W_{i}$ and by period. Included are the mean and standard deviation of the number of trips, the fraction in each of the four income categories (less than 17,000 guilders, between 17,000 and 24,000 guilders, between 24,000 and 38,000 guilders, and more than 38,000 guilders), and the fraction living in a central city. The first row gives the summary statistics for the first wave for those individuals who stayed in the sample for both waves. The second row gives the summary statistics for the first wave for individuals who dropped out of the sample after the first wave. The third row gives t-statistics for the difference in means between the two subsamples, corresponding to the MCAR null hypothesis that the willingness to respond $W_{i}$ is independent of the number of trips in both periods, the indicators for the income categories and the city indicator.

The average number of trips in the periods 1 and 2, computed from the unbalanced

\footnotetext{
${ }^{5}$ Again we ignore the initial nonresponse in the refreshment sample.
} 
panel only, is 55.0 and 54.9, respectively, an indication that travel demand has not changed. This is confirmed by the refreshment sample, as Ridder (1992) shows that the decrease in the refreshment sample average is due to differences in the sampling fractions in the strata. However, in the balanced panel there is a (statistically significant) decrease in the average number of trips from 61.8 to 54.9. Moreover, the households that stay in the panel make on average (significantly) more trips than households that drop out after the first wave. Hence, the probability of attrition is negatively correlated with the time average of the number of trips and positively correlated with an increase after the first wave.

The fourth row gives the statistics for the individuals who stayed in the panel in both periods, and the fifth row gives the results for the refreshment sample. For the last group we do not know the value of $W_{i}$. The last row reports t-statistics for the difference in means in the two subsamples. Here the implicit MCAR null hypothesis is that both the design variable $A_{i}$ and the willingness to respond $W_{i}$ are independent of all the other variables in the model. Both sets of t-statistics clearly demonstrate that the data are not missing completely at random, and therefore that using only the complete panel with $D_{i 1}=D_{i 2}=1$ may be very misleading. These tests do not reflect on the adequacy of the MAR and HW assumptions.

The two key variables, number of trips and income, are both characterized by a high degree of persistence. The correlation between first and second period values of the number of trips for the subsample who stays in the panel in both periods is 0.79 . The fraction of individuals in this subsample who stays in the same income category is 0.72 .

\subsection{The Model}

We make the following modelling assumptions, using $T_{1 i}$ and $T_{2 i}$ to denote the number of trips per household for the first and second period, $Y_{1 i}$ and $Y_{2 i}$ to denote household income for the first and second period, and $C_{i}$ to denote whether the household lives in a city. Conditional on living in a city, the joint distribution of the logarithm of household income in both periods and the logarithm of the number of trips in both periods (adding one for 
Table 5: Summary Statistics By Period

\begin{tabular}{|c|c|c|c|c|c|c|c|c|c|c|}
\hline Period & $A_{i}$ & $W_{i}$ & $\begin{array}{l}\text { Sample } \\
\text { Size }\end{array}$ & $\begin{array}{r}\text { Nur } \\
\text { of } r \\
\text { mean } \\
\end{array}$ & $\begin{array}{l}\text { aber } \\
\text { rips } \\
\text { (s.d.) }\end{array}$ & $\begin{aligned} & \text { Earn1 } \\
&< 17,000 \\
&\end{aligned}$ & $\begin{aligned} & \text { Earn2 } \\
& \geq 17,000 \\
&< 24,000 \\
&\end{aligned}$ & $\begin{aligned} & \text { Earn3 } \\
& \geq 24,000 \\
&< 38,000 \\
&\end{aligned}$ & $\begin{array}{c}\text { Earn } 4 \\
\geq 38,000\end{array}$ & City \\
\hline 1 & 0 & 1 & 1031 & 61.8 & $(34.9)$ & 0.14 & 0.21 & 0.35 & 0.29 & 0.07 \\
\hline 1 & 0 & 0 & 733 & 45.5 & $(33.2)$ & 0.25 & 0.23 & 0.28 & 0.24 & 0.14 \\
\hline \multicolumn{4}{|c|}{ t-test for diff. of means } & 9.9 & & 14.0 & 2.4 & 7.7 & 5.4 & 14.3 \\
\hline 2 & 0 & 1 & 1031 & 54.9 & $(30.8)$ & 0.11 & 0.20 & 0.38 & 0.30 & 0.07 \\
\hline 2 & 1 & - & 656 & 51.8 & $(32.9)$ & 0.15 & 0.25 & 0.35 & 0.25 & 0.27 \\
\hline \multicolumn{4}{|c|}{ t-test for diff. of means } & 1.9 & & 6.9 & 5.6 & 2.6 & 5.1 & 25.1 \\
\hline
\end{tabular}

each household to avoid problems with the less than one percent of the total number of observations with zero trips in the survey week), is assumed to be multivariate normal:

$$
\left(\begin{array}{c}
\ln \left(T_{1 i}+1\right) \\
\ln Y_{1 i} \\
\ln \left(T_{2 i}+1\right) \\
\ln Y_{2 i}
\end{array}\right) \mid \alpha, \mu, \Sigma, C_{i} \sim \mathcal{N}\left(\mu_{0}+\mu_{1} \cdot C_{i}, \Sigma\right)
$$

We also assume that, conditional on first and second period income and number of trips, the probability of attrition has a logistic form:

$$
\begin{aligned}
\operatorname{Pr}\left(W_{i}\right. & \left.=1 \mid T_{1 i}, Y_{1 i}, T_{2 i}, Y_{2 i}, C_{i}, \alpha, \mu, \Sigma\right) \\
& =\frac{\exp \left(\alpha_{0}+\alpha_{1} \cdot \ln \left(T_{1 i}+1\right)+\alpha_{2} \cdot \ln Y_{1 i}+\alpha_{3} \cdot \ln \left(T_{2 i}+1\right)+\alpha_{4} \cdot \ln Y_{2 i}+\alpha_{5} \cdot C_{i}\right)}{1+\exp \left(\alpha_{0}+\alpha_{1} \cdot \ln \left(T_{1 i}+1\right)+\alpha_{2} \cdot \ln Y_{1 i}+\alpha_{3} \cdot \ln \left(T_{2 i}+1\right)+\alpha_{4} \cdot \ln Y_{2 i}+\alpha_{5} \cdot C_{i}\right)}
\end{aligned}
$$

To create imputed values for the missing data, as well as to obtain draws from the posterior distribution of the parameters of the model, we use Markov Chain Monte Carlo (MCMC) methods (Geman and Geman, 1984; Gelfand, Hills, Racine-Poon, and Smith, 1990; Gelman and Rubin, 1992; Tanner, 1993) and in particular the DA algorithm developed by Tanner and Wong (1987). Recent economic applications include Albert and Chib (1993), 
Lancaster (1995), Geweke and Keane (1997), and Chamberlain and Hirano (1997). The specifics for the algorithm used here are discussed in Appendix B.

Given the imputed data sets we estimate the quantities of interest, e.g., regression coefficients defined in terms of the complete data sets. The approximate variances of the estimates of the regression coefficients are obtained by adding the average complete data variance and the variance of the estimates over the imputed data sets. See Rubin (1987) for details.

\subsection{The Results}

We estimate eight versions of the model. The versions differ by the missing data model, MCAR, MAR, HW or AN, and by the data set used, complete panel, panel or panel and refreshment sample. Given a specific model for the missing data process we create a number of imputed data sets. The primary interest here is in estimates of the regression coefficients in the regression of the change in log of the number of trips on the change in the log of earnings:

$$
\ln \left(T_{2 i}+1\right)-\ln \left(T_{1 i}+1\right)=\beta_{0}+\beta_{1} \cdot\left(\ln Y_{2 i}-\ln Y_{1 i}\right)+\varepsilon_{i}
$$

We focus on the estimate of $\beta_{1}$, the income elasticity of the number of trips.

For comparison the regression coefficient on the first period logarithm of earnings in the regression of the number of trips on the first period logarithm of number of trips using the complete data subsample with $A_{i}=2, W_{i}=1$, using the MCAR model to impute the actual level of earnings, is 0.63 with a standard error of 0.13 .

Table 7 gives the posterior means and standard deviations for the parameters of the probability of the willingness to respond.

\section{Conclusion}

Panel data sets can provide a much richer set of variables than cross-sections, but they often are subject to more severe missing data problems. Adding a sample consisting of new units randomly drawn from the original sample as replacements for units who have dropped 
Table 6: Estimates of Income Elasticity of Travel

\begin{tabular}{c|cccccc} 
Model & \multicolumn{2}{c}{$\begin{array}{c}\text { Complete Panel } \\
\text { est. }\end{array}$} & s.e. & est. & Panel & \multicolumn{2}{c}{ Panel and Refreshment Sample } \\
& & & & & est. & s.e. \\
\hline MCAR & 0.130 & $(0.064)$ & 0.139 & $(0.073)$ & 0.136 & $(0.073)$ \\
MAR & & 0.135 & $(0.073)$ & 0.139 & $(0.073)$ \\
HW & & 0.120 & $(0.070)$ & 0.134 & $(0.066)$ \\
AN & & & & 0.213 & $(0.145)$
\end{tabular}

out of the panel, a so-called refreshment sample, can be helpful in mitigating the effects of attrition, both by allowing for estimation of richer models and by making estimation of conventional models more precise. In this paper we have developed a family of models to incorporate the presence of refreshment samples and demonstrate in an application to a Dutch data set on travel behaviour that such models can lead to substantially different results than models assuming the missing data process is ignorable or conventional econometric models for panel data with attrition. 
Table 7: Posterior Means and Standard Deviations of Parameters of WillINGNESS TO RESPOND

\begin{tabular}{|c|c|c|c|c|c|c|c|c|c|c|c|}
\hline \multirow[t]{2}{*}{ model } & \multirow[t]{2}{*}{ data } & \multicolumn{2}{|c|}{$\ln \left(T_{i 1}+1\right)$} & \multicolumn{2}{|c|}{$\ln \left(Y_{i 1}\right)$} & \multicolumn{2}{|c|}{$\ln \left(T_{i 2}+1\right)$} & \multicolumn{2}{|c|}{$\ln \left(Y_{i 2}\right)$} & \multicolumn{2}{|c|}{$C_{i}$} \\
\hline & & mean & s.d. & mean & s.d. & mean & s.d. & mean & s.d. & mean & s.d. \\
\hline MAR & panel & 0.70 & $(0.08)$ & 0.03 & $(0.11)$ & 0 & - & 0 & - & -0.39 & $(0.14)$ \\
\hline MAR & all & 0.70 & $(0.08)$ & 0.04 & $(0.11)$ & 0 & - & 0 & - & -0.39 & $(0.14)$ \\
\hline HW & panel & 0 & - & 0 & - & 1.30 & $(0.16)$ & -0.15 & $(0.21)$ & -0.31 & $(0.16)$ \\
\hline HW & all & 0 & - & 0 & - & 0.85 & $(0.14)$ & 0.06 & $(0.18)$ & -0.38 & $(0.15)$ \\
\hline $\mathrm{AN}$ & all & 3.20 & $(0.37)$ & 0.15 & $(0.59)$ & -3.48 & $(0.50)$ & 0.23 & $(0.79)$ & -0.49 & $(0.19)$ \\
\hline
\end{tabular}

\section{Appendix A: Proofs}

\section{Proof of Theorem 1}

We can rewrite equations (7)-(10) as

$$
\begin{aligned}
& \alpha_{0}=g^{-1}\left(\left(1-q_{01}\right) r_{01} /\left(\left(1-q_{01}\right) r_{01}+\left(1-\hat{q}_{00}\right) r_{00}\right)\right) \\
& \alpha_{0}+\alpha_{1}=g^{-1}\left(\left(1-q_{11}\right) r_{11} /\left(\left(1-q_{11}\right) r_{11}+\left(1-\hat{q}_{10}\right) r_{10}\right)\right) \\
& \alpha_{0}+\alpha_{2}=g^{-1}\left(q_{01} r_{01} /\left(q_{01} r_{01}+\hat{q}_{00} r_{00}\right)\right) \\
& \alpha_{0}+\alpha_{1}+\alpha_{2}=g^{-1}\left(q_{11} r_{11} /\left(q_{11} r_{11}+\hat{q}_{10} r_{10}\right)\right)
\end{aligned}
$$

Eliminating $\alpha_{0}, \alpha_{1}$ and $\alpha_{2}$ leaves the restriction $h\left(\hat{q}_{00}, \hat{q}_{10}\right)=0$ where

$$
\begin{aligned}
h\left(q_{10}, q_{00}\right)= & g^{-1}\left(\frac{q_{11} r_{11}}{q_{11} r_{11}+q_{10} r_{10}}\right)+g^{-1}\left(\frac{\left(1-q_{01}\right) r_{01}}{\left(1-q_{01}\right) r_{01}+\left(1-q_{00}\right) r_{00}}\right) \\
& -g^{-1}\left(\frac{q_{01} r_{01}}{q_{01} r_{01}+q_{00} r_{00}}\right)-g^{-1}\left(\frac{\left(1-q_{11}\right) r_{11}}{\left(1-q_{11}\right) r_{11}+\left(1-q_{10}\right) r_{10}}\right) .
\end{aligned}
$$


Because of continuity of $h(\cdot, \cdot)$, and because $h(\cdot, \cdot)$ is increasing in $q_{00}$ and decreasing in $q_{10}$, this restriction defines an implicit function $\bar{q}_{10}\left(q_{00}\right)$ with the following properties:

$$
\frac{\partial \bar{q}_{10}}{\partial a}(a)>0, \quad \lim _{a \downarrow 0} \bar{q}_{10}(a)=0, \quad \text { and } \quad \lim _{a \uparrow 0} \bar{q}_{10}(a)=1 .
$$

Now consider the restriction (11). It defines a function

$$
\tilde{q}_{10}(a)=\frac{q_{00} r_{00}+q_{10} r_{10}-a r_{00}}{r_{10}}
$$

with the properties

$$
\frac{\partial \tilde{q}_{10}}{\partial a}(a)<0, \quad \tilde{q}_{10}(0)>0, \quad \text { and } \quad \tilde{q}_{10}(1)<1 .
$$

Hence there is a unique value $\hat{q}_{00}$ ) solving

$$
\bar{q}_{10}(a)=\tilde{q}_{10}(a)
$$

and $\hat{q}_{10}=\bar{q}_{10}\left(\hat{q}_{00}\right) . \square$.

The proof for Theorem 2 consists of three parts. First we prove two lemmas. The first lemma states that the solution $\hat{f}\left(z_{1}, z_{2}, x\right)$ can be characterized as the solution to a different problem. The second lemma states that the solution to the second problem is unique. Then we put these two results together. The discrete points of the support of the joint distribution of $\left(Z_{i 1}, Z_{i 2}\right)$ are organized in a sequence $\left\{\left(z_{1 k}, z_{2 k}\right)\right\}_{k=1}^{K}$. For a number of elements in this sequence $z_{1 k}=z_{1}$, or $z_{2 k}=z_{2}$ for that matter.

Lemma 1 Let $f\left(z_{1}, z_{2}\right)$ be the joint probability function of $\left(Z_{i 1}, Z_{i 2}\right)$, let $p\left(z_{1}, z_{2}\right)$ be the conditional probability that $W_{i}=1$ given $\left(Z_{i 1}, Z_{i 2}\right)$, and let $0<p\left(z_{1}, z_{2}\right)<1$ for all $\left(z_{1}, z_{2}\right)$ in the support of $\left(Z_{i 1}, Z_{i 2}\right)$. Let $g(\cdot)$ be a continuous, increasing function with $\lim _{a \rightarrow-\infty} g(a)=0$, and $\lim _{a \rightarrow \infty} g(a)=1$. Let $\left\{\left(z_{1 k}, z_{2 k}\right)\right\}_{k=1}^{K}$ be the support of $\left(Z_{i 1}, Z_{i 2}\right)$, and let

$$
\begin{aligned}
& q=\operatorname{Pr}\left(W_{i}=1\right)=\sum_{k=1}^{K} f\left(z_{1 k}, z_{2 k}\right) \cdot p\left(z_{1 k}, z_{2 k}\right), \\
& \pi_{z_{1} \cdot}=\operatorname{Pr}\left(Z_{i 1}=z_{1}\right)=\sum_{z_{2}} f\left(z_{1}, z_{2}\right),
\end{aligned}
$$




$$
\pi_{z_{2}}=\operatorname{Pr}\left(Z_{i 2}=z_{2}\right)=\sum_{z_{1}} f\left(z_{1}, z_{2}\right)
$$

and

$$
\pi_{k \mid 1}=\operatorname{Pr}\left(Z_{i 1}=z_{1 k}, Z_{i 2}=z_{2 k} \mid W_{i}=1\right)=f\left(z_{1 k}, z_{2 k}\right) \cdot p\left(z_{1 k}, z_{2 k}\right) / q
$$

Then if there is a set of functions $\hat{f}\left(z_{1}, z_{2}\right), k_{1}(z)$ and $k_{2}(z)$ and a constant $k_{0}$ such that for some $\left(\bar{z}_{1}, \bar{z}_{2}\right)$ in the support of $\left(Z_{i 1}, Z_{i 2}\right)$ :

(i), $k_{1}\left(\bar{z}_{1}\right)=0, k_{2}\left(\bar{z}_{2}\right)=0$,

(ii), $\sum_{z_{2}} \hat{f}\left(z_{1}, z_{2}\right)=\sum_{z_{2}} f\left(z_{1}, z_{2}\right)$,

(iii), $\sum_{z_{1}} \hat{f}\left(z_{1}, z_{2}\right)=\sum_{z_{1}} f\left(z_{1}, z_{2}\right)$,

(iv),

$$
\hat{f}\left(z_{1}, z_{2}\right)=f\left(z_{1}, z_{2}\right) \cdot \frac{p\left(z_{1}, z_{2}\right)}{g\left(k_{0}+k_{1}\left(z_{1}\right)+k_{2}\left(z_{2}\right)\right)},
$$

then

$$
p_{k}=\hat{f}\left(z_{1 k}, z_{2 k}\right)
$$

satisfies the first order conditions for a solution to the constrained maximization problem

$$
\begin{gathered}
\max _{p_{1}, \ldots, p_{K} \mid \pi_{k \mid 1} \cdot q<p_{k} \leq 1} \sum_{k=1}^{K} \pi_{k \mid 1} \cdot h\left(p_{k} / \pi_{k \mid 1}\right), \quad \text { subject to } \sum_{k=1}^{K} p_{k} \cdot 1\left\{z_{1 k}=z_{1}\right\}-\pi_{z_{1}}, \\
\sum_{k=1}^{K} p_{k} \cdot 1\left\{z_{2 k}=z_{2}\right\}-\pi_{\cdot z_{2}}, \quad \text { and } \sum_{k=1}^{K} p_{k}=1,
\end{gathered}
$$

where,

$$
h(a)=\left\{\begin{array}{cr}
-\int_{a}^{2 q} g^{-1}(q / s) d s & q<a<2 q \\
\int_{2 q}^{a} g^{-1}(q / s) d s & 2 q \leq a,
\end{array}\right\}
$$

and $h(a)$ not defined for $a \leq q$.

\section{Proof of Lemma 1:}

The argument consists of showing that $p_{k}=\hat{f}\left(z_{1 k}, z_{2 k}\right)$ solves the first order conditions for 
a solution to the maximization program, with the following Lagrange multipliers. For the restriction

$$
\sum_{k} p_{k} \cdot 1\left\{z_{1 k}=z_{1}\right\}-\pi_{z_{1}},
$$

the Lagrange multiplier is $\lambda_{z_{1}}$. The number of restrictions is equal to the number of points of support of $Z_{i 1}, K_{1}$, minus 1 . The omitted value is denoted by $\bar{z}_{1}$. For the restriction

$$
\sum_{k} p_{k} \cdot 1\left\{z_{2 k}=z_{2}\right\}-\pi \cdot z_{2},
$$

the Lagrange multiplier is $\gamma_{z_{2}}$. The number of restrictions equals the number of points of support of $Z_{i 2}, K_{2}$, minus 1 , with the omitted value denoted by $\bar{z}_{2}$. For the adding up restriction the Lagrange multiplier is $\delta$.

The first order condition for $p_{k}$ is:

$$
h^{\prime}\left(p_{k} / \pi_{k \mid 1}\right)-\delta-\sum_{z_{1}} \lambda_{z_{1}} \cdot 1\left\{z_{1 k}=z_{1}\right\}-\sum_{z_{2}} \gamma_{z_{2}} \cdot 1\left\{z_{2 k}=z_{1}\right\}=0 .
$$

By assumption the derivative of $h^{\prime}(\cdot)$ is invertible, with the inverse equal to $q / g(\cdot)$, so that the solution for $p_{k}$ is

$$
\begin{aligned}
p_{k}= & \pi_{k \mid 1} \cdot\left(h^{\prime}\right)^{-1}\left(\delta+\sum_{z_{1}} \lambda_{z_{1}} \cdot 1\left\{z_{1 k}=z_{1}\right\}+\sum_{z_{2}} \gamma_{z_{2}} \cdot 1\left\{z_{2 k}=z_{2}\right\}\right) \\
& =\pi_{k \mid 1} \cdot \frac{q}{g\left(\delta+\sum_{z_{1}} \lambda_{z_{1}} \cdot 1\left\{z_{1 k}=z_{1}\right\}+\sum_{z_{2}} \gamma_{z_{2}} \cdot 1\left\{z_{2 k}=z_{2}\right\}\right)} \\
& =\pi_{k \mid 1} \cdot \frac{q}{g\left(k_{0}+k_{1}\left(z_{1 k}\right)+k_{2}\left(z_{2 k}\right)\right)} .
\end{aligned}
$$

with $k_{1}, k_{2}$, and $k_{0}$ defined as $k_{1}\left(z_{1 k}\right)=\sum_{z_{1}} \lambda_{z_{1}} \cdot 1\left\{z_{1 k}=z_{1}\right\}, k_{2}\left(z_{2 k}\right)=\sum_{z_{2}} \gamma_{z_{2}} \cdot 1\left\{z_{2 k}=z_{2}\right\}$, and $k_{0}=\delta$.

If we substitute this solution in the restrictions, we obtain a system of $K_{1}+K_{2}-1$ equations in the same number of unknowns: $k_{0}$ and the values of the functions $k_{1}, k_{2}$ on the support of $Z_{i 1}, Z_{i 2}$, except $\bar{z}_{1}, \bar{z}_{2}$

$$
\pi_{z_{1},}-\sum_{k=1}^{K} \frac{q \pi_{k \mid 1}}{g\left(k_{0}+k_{1}\left(z_{1}\right)+k_{2}\left(z_{2 k}\right)\right)} 1\left\{z_{1 k}=z_{1}\right\}=0
$$




$$
\begin{aligned}
& \pi_{. z_{2}}-\sum_{k=1}^{K} \frac{q \pi_{k \mid 1}}{g\left(k_{0}+k_{1}\left(z_{1 k}\right)+k_{2}\left(z_{2}\right)\right)} 1\left\{z_{2 k}=z_{2}\right\}=0 \\
& 1-\sum_{k=1}^{K} \frac{q \pi_{k \mid 1}}{g\left(k_{0}+k_{1}\left(z_{1 k}\right)+k_{2}\left(z_{2 k}\right)\right)}=0
\end{aligned}
$$

Because $0<p\left(z_{1}, z_{2}\right)<1$, the variables in this system are bounded. If we let $k_{1}\left(z_{1}\right), k_{2}\left(z_{2}\right)$, $k_{0}$ increase, then the left-hand sides of the equations become nonnegative for a finite value of these variables, and irrespective of the values taken by the other (bounded) variables. If we let these variables decrease, then the left-hand sides become nonpositive, again for a finite value and irrespective of the values taken by the other (bounded) variables. Because the left-hand sides are continuous functions and there exists a bounded set such that these functions are nonnegative and nonpositive on the boundaries, we can invoke a fixed point theorem to show that the system of equations indeed has a (bounded) solution. Substitution of this solution in the equation for $p_{k}$ gives the desired result. $\square$.

Lemma 2 Let $\left\{z_{1 k}, z_{2 k}\right\}_{k=1}^{K}$ be the support of a pair of discrete random variables with probability $0<\pi_{k}<1$ for $k=1, \ldots, K$, and let $p\left(z_{1}, z_{2}\right)$ be a function such that $0<p\left(z_{1 k}, z_{2 k}\right)<1$ for all $k=1, \ldots, K$ with $q=\sum_{k} p\left(z_{1 k}, z_{2 k}\right) \cdot \pi_{k}$. Let $\pi_{z_{1}}=\sum_{k} \pi_{k} \cdot 1\left\{z_{1 k}=z_{1}\right\}$, and $\pi_{z_{2}}=\sum_{k} \pi_{k} \cdot 1\left\{z_{2 k}=z_{2}\right\}$. Furthermore, let $\pi_{k \mid 1}=\pi_{k} \cdot p\left(z_{1 k}, z_{2 k}\right) / q$. Finally let $h(\cdot)$ be any function defined on $(q, \infty)$ such that the inverse of the derivative of $h(\cdot)$ is equal to $q / g(a)$, for some increasing and continuous function $g(a)$ with $\lim _{a \rightarrow-\infty} g(a)=0$, and $\lim _{a \rightarrow \infty} g(a)=1$.

Then the optimization program

$$
\begin{gathered}
\max _{p_{1}, \ldots, p_{K} \mid q \pi_{k \mid 1}}<p_{k} \leq 1 \sum_{i=1}^{K} \pi_{k \mid 1} \cdot h\left(p_{k} / \pi_{k \mid 1}\right), \quad \text { subject to } \sum_{j} p_{j} \cdot 1\left\{\kappa_{j}=x\right\}-\pi_{z_{1}}, \\
\sum_{j} p_{j} \cdot 1\left\{\gamma_{j}=y\right\}-\pi_{\cdot z_{2}}, \quad \text { and } \sum p_{k}=1
\end{gathered}
$$

for all $z_{1}$ in the support of $Z_{i 1}$, and for all $z_{2}$ in the support of $Z_{i 2}$, has a unique solution. 


\section{Proof of Lemma 2:}

First consider the function $h(\cdot)$. Its second derivative is negative. In addition, $\lim _{a \downarrow q} h^{\prime}(a)=$ $\infty$, and $\lim _{a \rightarrow \infty} h^{\prime}(a)=-\infty$. Hence $h(\cdot)$ is bounded from above by some number $\bar{h}$.

Hence we are maximizing a concave function over a convex set. If the set over which the function is maximized were compact, this would guarantee the existence of a unique solution. However, the restriction $p_{k}>q \pi_{k \mid 1}$ implies the set is not compact. There are two possibilities. If the limit $a \downarrow q h(a)=\underline{h}>-\infty$, we can extend the definition of $h(\cdot)$ and maximize the function over a compact set. If the limit $a \downarrow q$ diverges, we can restrict the set of $p_{k}$ to those such that the objective function is greater than $c-\varepsilon$, where $c$ is the value at $p_{k}=\pi_{k \mid 1}$. This set will then be compact and the corresponding solution will be unique and in the interior.

\section{Proof of Theorem 2:}

For a given value for $x$, we can apply Lemma's 1 and 2 to prove the existence and uniqueness of $\hat{f}\left(z_{1}, z_{2}, x\right), k_{0}(x), k_{1}\left(z_{1}, x\right)$ and $k_{2}\left(z_{2}, x\right)$.

\section{Appendix B: MCMC Algorithms}

Although the specific details of the MCMC simulations discussed below depend on the particular models used for the conditional distribution of $\left(T_{1 i}, Y_{1 i}, T_{2 i}, Y_{2 i}\right)$ given $C_{i}$, and the conditional probability $\operatorname{Pr}\left(W_{i}=1 \mid T_{1 i}, Y_{1 i}, T_{2 i}, Y_{2 i}, C_{i}\right)$, for most conventional models MCMC methods will be easy to implement. In our implementation, the chains consist of six steps, the first four dealing with imputing the missing data given current parameter values, and the last two drawing from the posterior distributions of the parameters given imputed and observed data.

First, given initial values of the parameters, we impute the missing values for $T_{2 i}$ and $Y_{2 i}$ for units with $A_{i}=0$ and $W_{i}=0$, conditioning on $T_{1 i}, Y_{1 i}, C_{i}$ and $W_{i}=0$. We implement this by drawing from the conditional distribution of $\ln \left(T_{2 i}+1\right)$ and $\ln Y_{2 i}$ given $T_{1 i}, Y_{1 i}$ and $C_{i}$, which is bivariate normal, and rejecting draws with probability equal to 
$\operatorname{Pr}\left(W_{i}=1 \mid T_{1 i}, Y_{1 i}, T_{2 i}^{\text {imputed }}, Y_{2 i}^{\text {imputed }}, C_{i}\right)$.

Second, we imputed values for $T_{1 i}$ and $Y_{1 i}$ for units with $A_{i}=1$. The conditional distribution of $\ln \left(T_{1 i}+1\right)$ and $\ln Y_{1 i}$ given $T_{2 i}, Y_{2 i}, C_{i}$ and the parameters is bivariate normal and straightforward to draw from.

In third step we draw, for units with $A_{i}=1$, given parameters, $T_{2 i}, Y_{2 i}, C_{i}$ and given the previously imputed values $T_{1 i}^{\text {imputed }}$ and $Y_{1 i}^{\text {imputed }}$, the attrition indicator $W_{i}$ using a binomial distribution.

In the fourth step we impute earnings $Y_{1 i}$ for units with $D_{i 1}=1$ and $Y_{2 i}$ for units with $D_{i 2}=1$ given the observed indicators for the four ranges, the observed or imputed willingness to respond $W_{i}$, the other variables and the parameters. Two methods were used to impute the continuous earnings variable. In one method unrestricted normally distributed random variables are drawn without conditioning on the observed range or on $W_{i}$. These draws are then rejected if they are outside the appropriate range, and also rejected with a probability depending on the value of $W_{i}$. This simple method can be computationally very burdensome and lead to many rejected draws. A second method was therefore used if the first one did not lead to an acceptable draw with 30 attempts. In the second method a piecewise linear approximation to the normal distribution inside the appropriate range was used with the draws rejected at an appropriate rate to generate draws from the appropriate truncated normal distribution, whose draws were then rejected with some probability depending on the value of $W_{i}$. See Gelman, Carlin, Rubin, Stern (1995) who call this method "trapezoidal approximation followed by rejection sampling", Ripley (1987), and Hammersley and Handscomb, (1964).

In the fifth step we draw from the posterior distribution of $\mu$ and $\Sigma$ given observed and imputed data. Given standard prior distributions these posterior distributions are straightforward to draw from. We use an improper, flat, prior distribution on all elements of $\mu=\left(\mu_{0}^{\prime}, \mu_{1}^{\prime}\right)^{\prime}$ and an improper prior distribution on $\Sigma$ proportional to $|\Sigma|^{-2}$.

Finally, in the sixth step, we draw from the posterior distribution of $\alpha$ given observed 
and imputed data, using the Metropolis-Hastings algorithm (Metropolis, Rosenbluth, Rosenbluth, Teller and Teller, 1953; Hastings, 1970). We assume prior independence of the components of $\alpha$, using normal prior distributions centered around zero with standard deviations equal to the square root of the average square of the corresponding variables. This leads to a prior standard deviation for $\alpha_{1}$ of 1 , for $\alpha_{2}$ of 4 , for $\alpha_{3}$ of 3 , for $\alpha_{4}$ of 4 , for $\alpha_{5}$ of 3 , and for $\alpha_{6}$ of 0.3 .

We first ran one long chain, and used this to draw overdispersed starting values for a number of independent chains. We then used the Gelman-Rubin (1992) criteria to monitor convergence of the chains. The first long chain used zero starting values for the slope coefficients because maximum likelihood estimates are difficult to obtain. 


\section{REFERENCES}

Abowd, J. M. , B. Crepon, F. Kramarz, A. Trognon, (1995) "A la Recherche des Moments Perdus: Covariance Models for Unbalanced Panels with Endogenous Death", NBER Working Paper No. 180, Cambridge, Mass.

Albert, And S. Chib, (1993), "Bayesian Inference via Gibbs Sampling of Autoregressive Time Series Subject to Markov Mean and Variance Shifts", Journal of Business and Economic Statistics 11, 1-15.

Baltagi, B., (1995), Econometric Analysis of Panel Data, New York: Wiley.

Brownstone, D., And R. Valetta, (1996), "Modeling Earnings Measurement Error: A Multiple Imputation Approach," Review of Economics and Statistics 78, 705-717.

Chamberlain, G., (1984), "Panel Data", in Griliches and Intrilligator, (eds.), Handbook of Econometrics, Vol I, North Holland.

Chamberlain, G., And K. Hirano, (1997), "Predictive Distributions Based on Longitudinal Earnings Data," mimeo, Harvard University.

Dempster, A. P., N. Laird, And D. B. Rubin, (1977), "Maximum Likelihood Estimation from Incomplete Data Using the EM Algorithm," Journal of the Royal Statistical Society, series B, 39:1-38 (with discussion).

Fitzgerald, J., P. Gottschalk, and R. Moffitt, (1996), "An Analysis of Sample Attrition in Panel Data: The Michigan Panel Study of Income Dynamics", manuscript, Department of Economics, Johns Hopkins University.

Gelfand, A., S. Hills, A. Racine-Poon, And A. Smith, (1990), "Illustration of Bayesian Inference in Normal Data Models Using Gibbs Sampling", Journal of the American Statistical Association, 85, 398-405.

Gelman, A., And D. B. Rubin, (1992), "Inference from Iterative Simulations Using Multiple Sequences", Statistical Science, 7, 457-511.

Geman, S., And D. Geman, (1984), "Stochastic Relaxation, Gibbs Distributions, and the 
Bayesian Restoration of Images", IEEE Transactions on Pattern Analysis and Machine Intelligence, 6, 721-741.

GeweKe, J., (1996), "Posterior Simulators in Econometrics," in D. Kreps and K. F. Wallis (eds.), Advances in Economics and Econometrics: Theory and Applications, Cambridge: Cambridge University Press, 1997. (Invited symposium paper, Econometric Society Seventh World Congress.)

Geweke, J., And M. Keane, (1997), “An Empirical Analysis of Income Dynamics Among Men in the PSID: 1968-1989," working paper, University of Minnesota.

Hammersley, J.M., And D.C. Handscomb, (1964), Monte Carlo Methods, New York: Wiley.

Hansen, M.H., W.H. Hurwitz, And W.G. Madow, (1966), Sample Survey Methods and Theory, vol. I and II, New York: Wiley.

Hastings, W. K., (1970), "Monte Carlo Sampling Methods Using Markov Chains and Their Applications", Biometrika, Vol. 57, 97-109.

Hausman, J. A., And D. A. Wise, (1979), "Attrition Bias in Experimental and Panel Data: The Gary Income Maintenance Experiment," Econometrica 47, 455-473.

Heckman, J. J., (1979), "Sample Selection Bias as a Specification Error," Econometrica 47, $153-61$.

Honore, B., (1992), "Trimmed LAD and Least Squares Estimation of Truncated and Censored Regression Models with Fixed Effects," Econometrica 60, 533-565.

Horowitz, J., And C. Manski, (1998), "Censoring of Outcomes and Regressors Due to Survey Nonresponse: Identification Using Weights and Imputations," Journal of Econometrics, Vol. 84, No 1, 37-58.

Hsiao, C., (1986), Analysis of Panel Data, Cambridge: Cambridge University Press.

Imbens, G., And J. Hellerstein, (1995), "Imposing Moment Restrictions from Auxiliary Data by Weighting", mimeo, Harvard University.

Kyriazidou, E., (1997), "Estimation of a Panel Data Sample Selection Model," Econometrica 
65, 1335-1364.

LAnCAster, T., (1995), "Exact Structural Inference in Optimal Job Search Models," Working Paper No. 95-3, Department of Economics, Brown University.

Little, R., (1994), "A Class of Pattern Mixture Models for Normal Missing Data Models", Biometrika, Vol. 81, No 3, 471-483.

Little, R. J. A., And D. B. Rubin, (1987), Statistical Analysis with Missing Data, New York: Wiley.

Little, R., And M. Wu, (1991), "Models for Contingency Tables with Known Margins When Target and Sampled Populations Differ, Journal of the American Statistical Association, Vol. 86., No. 413, 87-95.

Little, R., And Y.-X. WAng, (1996), "Pattern Mixture Models for Multivariate Incomplete Data with Covariates", Biometrics, Vol. 52, 98-111.

Manski, C., (1995), Identification Problems in the Social Sciences Cambridge: Harvard University Press.

Meurs, H. And G. Ridder, (1992), "Attrition and Response Effects in the Dutch Mobility Panel," working paper, University of Amsterdam.

Metropolis, N, A. Rosenbluth, M. Rosenbluth, A. Teller, and E. Teller, (1953), "Equations of State Calculations by Fast Computing Machines", Journal of Chemical Physics, Vol. 21, 1087-1091.

Moffitt, R., J. Fitzgerald, P. Gottschalk, (1997), "Sample Selection in Panel Data: The Role of Selection on Observables," manuscript, Department of Economics, Johns Hopkins University.

Nevo, A., (1997), "Using Weights to Adjust for Sample Selection," mimeo, Department of Economics, University of California at Berkeley.

Ridder, G., (1990), "Attrition in Multi-wave Panel Data", in Hartog, Ridder and Theeuwes (eds.), 45-68, Panel Data and Labor Market Studies, North Holland.

Ridder, G., (1992), “An Empirical Evaluation of Some Models for Non-random Attrition in 
Panel Data," Structural Change and Economic Dynamics 3:2, 337-355.

Ripley, (1987), Stochastic Simulation, New York: Wiley.

Rubin, D. B., (1976), "Inference and Missing Data," Biometrika 63, 581-592.

Rubin, D. B., (1987), Multiple Imputation, New York: Wiley.

Rubin, D. B., (1996), "Multiple Imputation After 18+ Years", (with discussion) Journal of the American Statistical Assocation, Vol 91, No. 434, 473-521.

TAnner, M., (1993) Tools for Statistical Inference, New York: Springer-Verlag.

Tanner, M. And W. Wong, (1990), "The Calculation of Posterior Distributions by Data Augmentation," (with discussion), Journal of the American Statistical Association, Vol. 82, No. $398,528-550$.

Verbeek, M. and T. Nijman, (1992), "Testing for Selectivity Bias in Panel Data Models," International Economic Review, Vol 33, No. 3, 681-703.

Wooldridge, J., (1995), "Selection Corrections for Panel Data Models under Conditional Mean Independence Assumptions," Journal of Econometrics, 68, 115-132. 